\title{
Effect of chitosan oligosaccharide and valine on growth, serum hormone levels and meat quality of broilers
}

\author{
Iftikhar Ahmed ${ }^{1 \#}$, Nabila Roohi ${ }^{1}$ \& Ayesha Roohi ${ }^{2}$ \\ ${ }^{1}$ Physiology / Endocrinology Laboratory, Department of Zoology, University of the Punjab, Quaid-e-Azam Campus, \\ Lahore-54590 \\ 2 Department of Chemistry Kinnaird College for Women, Lahore
}

(Submitted 14 May 2020; Accepted 19 November 2020; Published 6 January 2021)

\author{
See: http://creativecommons.org/licenses/by/4.0/za \\ Condition of use: The user may copy, distribute, transmit and adapt the work, but must recognize the authors and the South African \\ Journal of Animal Science.
}

\begin{abstract}
Chitosan oligosaccharide (COS) and valine (Val) supplementation will improve growth performance, carcass traits, hormonal profile and meat quality in broilers. To evaluate this hypothesis, based on a $2 \times 4$ factorial arrangement, 480 male broilers (Ross 708) were randomly placed in eight treatment groups for two levels of $\operatorname{COS}(\mathrm{C} 1: 100 \mathrm{mg} / \mathrm{kg}$, and $\mathrm{C} 2: 150 \mathrm{mg} / \mathrm{kg})$ and four levels of valine (V1: $0.57 \%, \mathrm{~V} 2: 0.72 \%, \mathrm{~V} 3$ : $0.87 \%$ and V4: $1.02 \%)$ with three replicates $(n=24)$ with 20 birds in each $\left(n_{i}=60 ; i=1,2,3, \ldots, 8\right.$.). The results showed that live bodyweight (BW), weight gain (WG), and carcass weight increased, and feed conversion ratio (FCR) decreased with increased supplemental levels of dietary Val at $\mathrm{C} 1$ and $\mathrm{C} 2$. Abdominal fat reduced linearly for both $\mathrm{COS}$ and $\mathrm{Val}$, with a higher reduction response value at $\mathrm{C} 2$ and V4. The serum levels of triiodothyronine $\left(\mathrm{T}_{3}\right)$, thyroxine $\left(\mathrm{T}_{4}\right)$, testosterone and insulin-like growth factor 1 (IGF-1) presented a linear effect for COS and Val. The proximate composition of breast and leg meat showed that crude fat content decreased linearly for COS and Val with higher reduction response values at V3 for both levels of COS. The ash content rose linearly with increasing concentration of Val at $\mathrm{C} 1$, but showed the highest value at V2 when birds were offered $\mathrm{C} 2$. There was an interaction between COS and Val for $\mathrm{T}_{3}, \mathrm{~T}_{4}, \mathrm{IGF}-1$, ash content and crude fat. In conclusion, supplementation exerted a significant influence on the growth performance, hormonal profile and meat composition of broilers.
\end{abstract}

Key words: abdominal fat, ash content, carcass, feed conversion ratio

\#Corresponding author: \&ifti758@gmail.com

\section{Introduction}

The widespread use of antibiotics as growth promoters had adverse effects on poultry and on antibiotic resistance in microbes. Another harmful aspect of such practices was that traces of antibiotics became part of animal meat and ultimately human food (Qu et al., 2019; Amirdahri et al., 2020). To avoid these undesirable consequences, poultry breeders and researchers are working to develop feed formulations that have potential antibiotic alternatives or at least to adopt an integrated approach to minimize toxicity and hazardous effects (Janardhana et al., 2009; Nazir et al., 2020). For this purpose, the inclusion of amino acids and prebiotics in poultry diets has been widely accepted as alternatives to antibiotics because of their growth- and health-promoting effects (Shi et al., 2005).

Chitosan oligosaccharide (COS) is an oligomer that is derived from chitin through de-acetylation and hydrolysis and has several biological properties that make it an attractive replacement for applications in medical and nutrition sciences. Chitosan oligosaccharide is non-toxic, biocompatible, biodegradable, and antifungal (Haque et al., 2017). The effect of COS in promoting an immune response has aroused interest, because COS could enhance the migratory activity of macrophages, the cells that play a vital role in the defence mechanisms of host immune responses by promoting the release of cytokines (Qureshi et al., 2000; Xiong et al., 2015). Research findings posited that chitosan had a significant influence on the improvement of average daily weight gain, and a decrease in the percentage of dissected abdominal fat, subcutaneous fat, inter-muscular fat depth, and the levels of triglycerides and cholesterol (Liu et el., 2008; Swiatkiewicz et al., 2015). 
Essential amino acids are fundamental dietary requirements for the growth and metabolism of all organisms. Among the nine essential amino acids there are three branched-chain amino acids (BCAAs), namely leucine, isoleucine and Val. The supplementation of specific amino acids in corn and soybean basal diets for poultry has gained importance among researchers in attempting to provide the least possible amount of crude protein in formulations of broiler feed (Liu et al., 2019; Sterling et al., 2003). Various dietary formulations have been employed in poultry practices to attain an ideal balance among amino acids in broiler diets (Aftab, 2009; Ayasan et al., 2009; Ayasan \& Okan, 2014). It has also been determined that Val is an important nutritional supplement in broiler diets to encourage growth performance and to induce health benefits (Duarte et al., 2014; Ferreira et al., 2016).

The use of COS could promote growth by increasing digestibility and enhancing microbial activity in the gut, whereas Val could enhance muscle protein synthesis (Miranda et al., 2015). Dietary formulations that contained various levels of crude protein supplemented with COS and Val would increase growth performance (Ospina-Rojas et al., 2014; Zhao et al., 2015) and carcass yield in broilers, and lead to an improvement in FCR and efficiency of meat production because broilers are a quick, accessible and cheap source of meat (Sedghi et al., 2015; Ospina-Rojas et al., 2020).

Based on these arguments, there is a need to consolidate the concept that COS and Val are growth promoters and could be used as alternatives to antibiotic growth promoters. Hence, the present study was contemplated to examine the hypothesis that COS and Val will accelerate growth, and improve the carcass characteristics, hormonal profile and proximate meat composition of broilers.

\section{Materials and Methods}

Ethical approval for this research was granted by the Advanced Studies and Research Board/Committee, University of the Punjab, Lahore, on 20 October 2018.

The present study was intended to improve the growth performance and meat quality of commercially important Ross 708 broilers during the period of growth up day 42 post hatch. For this purpose, based on a $2 \times 4$ factorial arrangement, a total of 480 broilers (one day old) were randomly placed in eight treatments for two levels of COS (C1: $100 \mathrm{mg} / \mathrm{kg}$ and C2: $150 \mathrm{mg} \mathrm{kg}$ ) and four levels of valine (V1: $0.57 \%, \mathrm{~V} 2: 0.72 \%$, V3: $0.87 \%$ and V4: $1.02 \%)$ with three replicates $(n=24)$ for each treatment $\left(n_{i}=60 ; I=1,2,3, \ldots, 8\right)$ comprising 20 birds each. The birds were fed a corn-soybean-based diet up to 20 days with all the recommended ingredients (Rostagno et al., 2011) and a calculated metabolized energy (ME) value of $3110 \mathrm{kcal} / \mathrm{kg}$ (Table 1). The experimental diets were prepared by adding various concentrations of COS and dietary Val at the expense of inert filler (sand).

The experimental birds were kept in three-tiered battery cages (with a floor space of $0.7 \mathrm{~m}^{2}$ for each bird), equipped with trough feeders and nipple drinkers, in a well-ventilated solid-walled poultry facility under standard poultry practices with lighting schedule of 20 hours light and 4 hours darkness. Throughout the research, the birds were examined physically and vaccinated accordingly. The experimental procedures were conducted at Government Post Graduate Islamia College Gujranwala, whereas the samples were preserved and analysed in the Physiology/Endocrinology Laboratory, Department of Zoology, University of the Punjab, Quaid-e-Azam Campus, Lahore. Meat quality parameters were analysed in the Animal Nutrition Research Laboratory at University of Veterinary and Animal Sciences (UVAS), Pattoki Campus.

The BW and feed intake (FI) data were recorded weekly on the 7th, 14th, 21st, 28th, 35th, and 42nd day. Data on carcass weight, abdominal fat, and the relative weights of liver, heart, spleen, gall bladder and gizzard were recorded on day 42 . Blood samples to evaluate hormonal parameters were acquired from one bird from each replicate $(n=24)$ at the age of 42 days. After serum isolation, tests for hormonal profile were performed with serological kits and analysed in COBAS model c111 (Roche Digitals, Basle, Switzerland).

Meat quality parameters for proximate meat composition were analysed with standard protocols and apparatus. Breast and leg samples were acquired from three birds for each treatment group, preserved and analysed chemically according to the official methods of analysis (AOAC, 2006). Dry matter and moisture content in meat samples were determined by drying at $105^{\circ} \mathrm{C}$ to constant weight, followed by desiccation. To estimate crude protein, crude lipid and ash content, Kjeldahl flask apparatus, Soxtherm extraction method and muffle furnace were used.

Statistical analysis of the data was performed by applying two-way factorial ANOVA, using the general linear model procedures of SPSS (IBM Corp., Armonk, New York, USA). For comparative analysis among treatments, Duncan's multiple range test (Duncan, 1955) was used and treatment means were considered different at $P<0.05$. 
Table 1 Ingredients and nutrient composition of the basal experimental diet for broilers

\begin{tabular}{|c|c|}
\hline Ingredients & Composition \\
\hline Corn & 67.30 \\
\hline Soybean meal, $44 \%$ crude protein & 14.30 \\
\hline Palm oil & 6.60 \\
\hline Fish meal, $71.7 \%$ crude protein & 3.00 \\
\hline Molasses & 3.00 \\
\hline Di-calcium phosphate & 1.55 \\
\hline Limestone & 1.30 \\
\hline $\mathrm{NaCl}$ & 0.50 \\
\hline Vitamin mineral premix ${ }^{1}$ & 0.20 \\
\hline I-lysine $\mathrm{HCl}$ & 0.70 \\
\hline DL methionine & 0.20 \\
\hline Threonine & 0.15 \\
\hline Filler (sand) & 1.20 \\
\hline Nutrients (\%) & \\
\hline Metabolizable energy (kcal/kg) & 3110 \\
\hline Crude protein & 20.18 \\
\hline Available phosphorus & 0.45 \\
\hline Calcium & 0.83 \\
\hline Potassium & 0.78 \\
\hline Sodium & 0.18 \\
\hline Chloride & 0.25 \\
\hline Crude fibre & 4.40 \\
\hline Linoleic acid & 1.00 \\
\hline Lysine \& & 1.22 \\
\hline Methionine + cysteine & 0.90 \\
\hline Standard ileal digestible methionine & 0.50 \\
\hline Standard ileal digestible cysteine & 058 \\
\hline Standard ileal digestible tryptophan & 0.15 \\
\hline Standard ileal digestible arginine & 0.95 \\
\hline Standard ileal digestible threonine & 0.69 \\
\hline Standard ileal digestible leucine & 1.12 \\
\hline Standard ileal digestible valine & 0.57 \\
\hline Standard ileal digestible isoleucine & 0.75 \\
\hline Standard ileal digestible phenylalanine & \\
\hline
\end{tabular}

\footnotetext{
${ }^{1}$ Vitamin $A: 12000 \mathrm{IU}$, vitamin $\mathrm{D}_{3}: 1500 \mathrm{IU}$, vitamin $\mathrm{E}: 30 \mathrm{mg}$, vitamin $\mathrm{K}_{3}: 5 \mathrm{mg}$, vitamin $\mathrm{B}_{1}: 3 \mathrm{mg}$, vitamin $\mathrm{B}_{2}: 6 \mathrm{mg}$, vitamin $B_{6}: 5 \mathrm{mg}$, vitamin $B_{12}: 0.03 \mathrm{mg}$, nicotinic acid amine: $40 \mathrm{mg}$, Di-calcium pantothenate: $10 \mathrm{mg}$, folic acid: 0.075 mg, choline: $370 \mathrm{mg}$, manganese: $85 \mathrm{mg}$, iron: $80 \mathrm{mg}$, copper: $8 \mathrm{mg}$, iodine: $0.5 \mathrm{mg}$, cobalt: $0.25 \mathrm{mg}$, selenium: $0.10 \mathrm{mg}$
}

\section{Results and Discussion}

Supplementation with COS did not produce significant effects on live BW, WG, FI and FCR. However, live BW, WG and FI increased when broiler diets were supplemented with increasing levels of $\mathrm{Val}(P<0.05)$. The birds were most efficient at V4. The interaction of COS and Val did not approach significance $(P>0.20)$ for any of these measures of performance. The results for growth performance attributes are presented in Table 2. 
Table 2 Live weight, weight gain, feed intake and feed conversion ratio of broilers at 42 days old when supplemented with various levels of chitosan oligosaccharide and valine

\begin{tabular}{|c|c|c|c|c|c|}
\hline \multirow{2}{*}{\multicolumn{2}{|c|}{ Treatments }} & \multicolumn{4}{|c|}{ Response variables } \\
\hline & & \multirow{2}{*}{$\begin{array}{l}\text { Live bodyweight, g } \\
2714.91 \pm 25.68\end{array}$} & \multirow{2}{*}{$\begin{array}{c}\text { Weight gain, g } \\
2673.25 \pm 25.40\end{array}$} & \multirow{2}{*}{$\begin{array}{l}\text { Feed intake, } g \\
4668.75 \pm 19.06\end{array}$} & \multirow{2}{*}{$\begin{array}{c}\text { Feed conversion } \\
1.75 \pm 0.01\end{array}$} \\
\hline C1 & & & & & \\
\hline \multirow[t]{5}{*}{$\mathrm{C} 2$} & & $2740.00 \pm 26.52$ & $2699.08 \pm 26.63$ & $4698.70 \pm 21.20$ & $1.74 \pm 0.01$ \\
\hline & V1 & $2635.50 \pm 24.56^{\mathrm{a}}$ & $2594.83 \pm 24.24^{a}$ & $4648.33 \pm 31.53^{a}$ & $1.79 \pm 0.01^{c}$ \\
\hline & V2 & $2680.50 \pm 16.08^{a}$ & $2638.83 \pm 16.12^{\mathrm{a}}$ & $4626.66 \pm 18.35^{\mathrm{a}}$ & $1.76 \pm 0.00^{b}$ \\
\hline & V3 & $2764.66 \pm 19.60^{b}$ & $2724.33 \pm 19.38^{b}$ & $4683.33 \pm 16.31^{\mathrm{ab}}$ & $1.72 \pm 0.01^{\mathrm{a}}$ \\
\hline & V4 & $2829.16 \pm 19.93^{c}$ & $2786.67 \pm 21.02^{b}$ & $4716.67 \pm 24.63^{b}$ & $1.70 \pm 0.01^{a}$ \\
\hline C1 & $\mathrm{V} 1$ & $2624.33 \pm 40.99$ & $2584.33 \pm 40.61$ & $4648.33 \pm 62.87$ & $1.79 \pm 0.01$ \\
\hline $\mathrm{C} 1$ & V2 & $2659.33 \pm 12.20$ & $2618.33 \pm 11.68$ & $4626.67 \pm 16.41$ & $1.76 \pm 0.00$ \\
\hline C1 & V3 & $2769.33 \pm 29.42$ & $2728.67 \pm 29.36$ & $4683.33 \pm 06.67$ & $1.71 \pm 0.02$ \\
\hline C1 & V4 & $2806.67 \pm 21.86$ & $2762.00 \pm 24.01$ & $4716.67 \pm 37.11$ & $1.70 \pm 0.00$ \\
\hline $\mathrm{C} 2$ & $\mathrm{~V} 1$ & $2646.67 \pm 34.80$ & $2605.67 \pm 34.23$ & $4628.33 \pm 30.32$ & $1.77 \pm 0.01$ \\
\hline $\mathrm{C} 2$ & V2 & $2701.67 \pm 26.39$ & $2659.33 \pm 27.24$ & $4673.33 \pm 29.48$ & $1.75 \pm 0.01$ \\
\hline $\mathrm{C} 2$ & V3 & $2760.00 \pm 32.15$ & $2720.00 \pm 31.58$ & $4710.00 \pm 33.29$ & $1.73 \pm 0.01$ \\
\hline $\mathrm{C} 2$ & V4 & $2851.67 \pm 31.67$ & $2811.33 \pm 31.99$ & $4783.33 \pm 23.33$ & $1.70 \pm 0.01$ \\
\hline
\end{tabular}

C1: chitosan oligosaccharide (COS) supplemented at $100 \mathrm{mg} / \mathrm{kg}, \mathrm{C} 2$ : COS supplemented at $150 \mathrm{mg} / \mathrm{kg}, \mathrm{V} 1: \mathrm{valine}$ supplemented at $0.57 \%, \mathrm{~V} 2$ : valine supplemented at $0.72 \%$, V3: valine supplemented at $0.87 \%$, and V4: valine supplemented at $1.02 \%$ of basal diet

Both carcass weight and its percentage increased linearly with increasing concentration of Val $(P$ $<0.05)$. Both abdominal fat and its percentage decreased linearly with the increase in supplemental Val $(P$ $<0.05)$ and decreased with increased COS. Again, the interaction between levels of COS supplementation and Val did not approach significance $(P>0.20)$. The effects of COS and Val on carcass weight and abdominal fat and their relative percentages are presented in Table 3.

The main effects of various levels of COS and Val and their interaction had no significant effects $(P$ $>0.05$ ) on the relative weights of liver, heart, spleen, gizzard and gall bladder (Table 4).

Dietary supplementation of COS and Val resulted in significant interactions that affected the levels of $T_{3}, T_{4}$ and IGF-1 (Table 5). The interaction effect on $T_{3}$ was due primarily to the marked effect of COS that was seen at V2, whereas the effects of COS at the other levels of Val were much less. For $T_{4}$, the interaction was due to an increasing effect at C1 as the level of Val supplementation rose, whereas at C2 the effect of Val supplementation was negligible. The interaction effect on IGF-1 was similar to that of $T_{4}$, except that at the high levels of COS and Val a marked depression was observed in the serum level. There was no significant effect of supplementation on thyroid stimulating hormone (TSH) $(P>0.05)$, though it showed a numerical increase with rising levels of supplemental COS and Val. Serum level of testosterone rose linearly $(P<0.05)$ with the increase in COS and more markedly with the rising levels of supplemental Val. However, there was no interaction between COS and Val that affected testosterone level.

Proximate analysis of the breast meat revealed that the meat became significantly drier as the level of Val supplementation increased (Table 6$)$. Supplementation did not affect $(P>0.05)$ crude protein content or the $\mathrm{pH}$ of the breast meat. The crude fat and ash contents were affected by the interaction of the levels of $\mathrm{COS}$ and Val. When $\mathrm{C} 1$ was provided in the diet, the crude fat content of the breast muscle decreased by $0.7 \%$ as the level of Val increased. However, it decreased by $1.2 \%$ when the broilers were fed C2. Likewise, the ash content of the breast muscle increased by $1.4 \%$ as the level of Val supplementation increased at C1, but it only increased by $0.7 \%$ at C2. 
Table 3 Weights and percentages of carcass and abdominal fat of broilers of broilers at 42 days old when supplemented with various levels of chitosan oligosaccharide and valine

\begin{tabular}{|c|c|c|c|c|c|}
\hline \multirow{2}{*}{\multicolumn{2}{|c|}{ Treatments }} & \multicolumn{4}{|c|}{ Response variables } \\
\hline & & \multirow{2}{*}{$\begin{array}{l}\text { Carcass weight, g } \\
1917.08 \pm 36.44\end{array}$} & \multirow{2}{*}{$\begin{array}{l}\text { Carcass, \% } \\
70.55 \pm 0.82\end{array}$} & \multirow{2}{*}{$\begin{array}{c}\text { Abdominal fat, } g \\
40.95 \pm 0.98^{\mathrm{a}}\end{array}$} & \multirow{2}{*}{$\begin{array}{c}\text { Abdominal fat, \% } \\
1.51 \pm 0.05^{\mathrm{a}}\end{array}$} \\
\hline $\mathrm{C} 1$ & & & & & \\
\hline \multirow[t]{5}{*}{ C2 } & & $1951.66 \pm 32.98$ & $71.19 \pm 0.76$ & $37.75 \pm 1.28^{\mathrm{b}}$ & $1.38 \pm 0.06^{b}$ \\
\hline & $\mathrm{V} 1$ & $1792.50 \pm 19.82^{c}$ & $68.01 \pm 0.43^{\mathrm{a}}$ & $44.18 \pm 0.86^{c}$ & $1.68 \pm 0.04^{c}$ \\
\hline & V2 & $1891.66 \pm 20.88^{b}$ & $70.56 \pm 0.61^{\mathrm{ab}}$ & $39.90 \pm 1.36^{\mathrm{b}}$ & $1.49 \pm 0.05^{b}$ \\
\hline & V3 & $1984.16 \pm 29.90^{\mathrm{a}}$ & $71.77 \pm 1.07^{\mathrm{b}}$ & $38.22 \pm 1.12^{b}$ & $1.38 \pm 0.04^{b}$ \\
\hline & V4 & $2069.16 \pm 25.01^{a}$ & $73.15 \pm 1.00^{\mathrm{b}}$ & $35.12 \pm 1.03^{a}$ & $1.24 \pm 0.04^{\mathrm{a}}$ \\
\hline C1 & V1 & $1768.33 \pm 28.92$ & $67.38 \pm 0.19$ & $44.73 \pm 1.07$ & $1.70 \pm 0.03$ \\
\hline C1 & V2 & $1866.67 \pm 29.62$ & $70.19 \pm 1.24$ & $42.13 \pm 0.74$ & $1.58 \pm 0.02$ \\
\hline $\mathrm{C} 1$ & V3 & $1980.00 \pm 55.07$ & $71.49 \pm 1.23$ & $39.97 \pm 1.44$ & $1.44 \pm 0.06$ \\
\hline $\mathrm{C} 1$ & V4 & $2053.33 \pm 29.48$ & $73.15 \pm 1.61$ & $36.97 \pm 1.05$ & $1.32 \pm 0.04$ \\
\hline $\mathrm{C} 2$ & $\mathrm{~V} 1$ & $1816.67 \pm 23.33$ & $68.63 \pm 0.71$ & $43.63 \pm 1.49$ & $1.65 \pm 0.08$ \\
\hline $\mathrm{C} 2$ & V2 & $1916.67 \pm 26.03$ & $70.94 \pm 0.42$ & $37.67 \pm 1.93$ & $1.39 \pm 0.07$ \\
\hline $\mathrm{C} 2$ & V3 & $1988.33 \pm 37.67$ & $72.04 \pm 2.02$ & $36.47 \pm 1.04$ & $1.32 \pm 0.05$ \\
\hline $\mathrm{C} 2$ & V4 & $2085.00 \pm 44.81$ & $73.11 \pm 1.55$ & $33.27 \pm 0.90$ & $1.17 \pm 0.03$ \\
\hline
\end{tabular}

C1: chitosan oligosaccharide (COS) supplemented at $100 \mathrm{mg} / \mathrm{kg}, \mathrm{C} 2$ : COS supplemented at $150 \mathrm{mg} / \mathrm{kg}$, V1: valine supplemented at $0.57 \%$, V2: valine supplemented at $0.72 \%$, V3: valine supplemented at $0.87 \%$, V4: valine supplemented at $1.02 \%$ of basal diet

${ }^{a-c}$ Within an effect on each response variable, values with a common superscript were not different at $P=0.05$

Table 4 Relative weights of liver, heart, spleen, gizzard, and gall bladder of broilers of broilers at 42 days old when supplemented with various levels of chitosan oligosaccharide and valine

\begin{tabular}{|c|c|c|c|c|c|c|}
\hline \multirow{2}{*}{\multicolumn{2}{|c|}{ Treatments }} & \multicolumn{5}{|c|}{ Response variables } \\
\hline & & \multirow{2}{*}{$\begin{array}{c}\text { Liver, \% } \\
2.22 \pm 0.04\end{array}$} & \multirow{2}{*}{$\begin{array}{c}\text { Heart, \% } \\
0.70 \pm 0.01\end{array}$} & \multirow{2}{*}{$\begin{array}{l}\text { Spleen, \% } \\
0.12 \pm 0.00\end{array}$} & \multirow{2}{*}{$\begin{array}{l}\text { Gizzard, \% } \\
1.20 \pm 0.02\end{array}$} & \multirow{2}{*}{$\begin{array}{c}\text { Gall bladder, \% } \\
0.12 \pm 0.00\end{array}$} \\
\hline $\mathrm{C} 1$ & & & & & & \\
\hline $\mathrm{C} 2$ & & $2.12 \pm 0.05$ & $0.69 \pm 0.01$ & $0.11 \pm 0.00$ & $1.24 \pm 0.02$ & $0.12 \pm 0.00$ \\
\hline & V1 & $2.27 \pm 0.07$ & $0.71 \pm 0.02$ & $0.12 \pm 0.00$ & $1.26 \pm 0.03$ & $0.13 \pm 0.01$ \\
\hline & V2 & $2.12 \pm 0.03$ & $0.70 \pm 0.01$ & $0.12 \pm 0.00$ & $1.24 \pm 0.03$ & $0.12 \pm 0.00$ \\
\hline & V3 & $2.17 \pm 0.07$ & $0.68 \pm 0.01$ & $0.11 \pm 0.00$ & $1.16 \pm 0.03$ & $0.12 \pm 0.00$ \\
\hline & V4 & $2.12 \pm 0.06$ & $0.69 \pm 0.01$ & $0.12 \pm 0.01$ & $1.24 \pm 0.03$ & $0.11 \pm 0.01$ \\
\hline $\mathrm{C} 1$ & V1 & $2.25 \pm 0.06$ & $0.70 \pm 0.03$ & $0.12 \pm 0.00$ & $1.21 \pm 0.02$ & $0.11 \pm 0.00$ \\
\hline $\mathrm{C} 1$ & V2 & $2.11 \pm 0.06$ & $0.69 \pm 0.02$ & $0.12 \pm 0.00$ & $1.29 \pm 0.03$ & $0.12 \pm 0.01$ \\
\hline $\mathrm{C} 1$ & V3 & $2.29 \pm 0.10$ & $0.69 \pm 0.01$ & $0.12 \pm 0.01$ & $1.13 \pm 0.05$ & $0.12 \pm 0.01$ \\
\hline $\mathrm{C} 1$ & V4 & $2.22 \pm 0.08$ & $0.71 \pm 0.01$ & $0.12 \pm 0.01$ & $1.19 \pm 0.05$ & $0.12 \pm 0.01$ \\
\hline $\mathrm{C} 2$ & $\mathrm{~V} 1$ & $2.29 \pm 0.15$ & $0.72 \pm 0.03$ & $0.12 \pm 0.00$ & $1.30 \pm 0.05$ & $0.14 \pm 0.00$ \\
\hline $\mathrm{C} 2$ & V2 & $2.13 \pm 0.00$ & $0.71 \pm 0.02$ & $0.12 \pm 0.00$ & $1.20 \pm 0.04$ & $0.12 \pm 0.00$ \\
\hline $\mathrm{C} 2$ & V3 & $2.04 \pm 0.05$ & $0.67 \pm 0.01$ & $0.11 \pm 0.01$ & $1.19 \pm 0.04$ & $0.12 \pm 0.00$ \\
\hline $\mathrm{C} 2$ & V4 & $2.02 \pm 0.04$ & $0.67 \pm 0.00$ & $0.11 \pm 0.00$ & $1.28 \pm 0.00$ & $0.11 \pm 0.00$ \\
\hline
\end{tabular}

C1: chitosan oligosaccharide (COS) supplemented at $100 \mathrm{mg} / \mathrm{kg}, \mathrm{C} 2$ : COS supplemented at $150 \mathrm{mg} / \mathrm{kg}$, V1: valine supplemented at $0.57 \%$, V2: valine supplemented at $0.72 \%$, V3: valine supplemented at $0.87 \%$, V4: valine supplemented at $1.02 \%$ of basal diet 
Table 5 Serum triiodothyronine, thyroxine, thyroid stimulating hormone, testosterone, and insulin-like growth factor 1 of 42 day-old broilers when supplemented with various levels of chitosan oligosaccharide and valine

\begin{tabular}{|c|c|c|c|c|c|c|}
\hline \multirow{2}{*}{\multicolumn{2}{|c|}{ Treatments }} & \multicolumn{5}{|c|}{ Response variables } \\
\hline & & \multirow{2}{*}{$\begin{array}{c}\mathrm{T}_{3}, \mathrm{ng} / \mathrm{ml} \\
5.25 \pm 0.23^{\mathrm{a}}\end{array}$} & \multirow{2}{*}{$\begin{array}{c}\mathrm{T}_{4}, \mu \mathrm{g} / \mathrm{dl} \\
1.74 \pm 0.09^{\mathrm{b}}\end{array}$} & \multirow{2}{*}{$\begin{array}{l}\mathrm{TSH}, \mu \mathrm{IU} / \mathrm{ml} \\
0.03 \pm 0.00\end{array}$} & \multirow{2}{*}{$\begin{array}{c}\text { Testosterone, } \mathrm{ng} / \mathrm{ml} \\
0.80 \pm 0.01^{\mathrm{b}}\end{array}$} & \multirow{2}{*}{$\begin{array}{l}\text { IGF-1, ng/ml } \\
3.61 \pm 0.07^{\mathrm{a}}\end{array}$} \\
\hline C1 & & & & & & \\
\hline \multirow[t]{5}{*}{$\mathrm{C} 2$} & & $4.50 \pm 0.16^{\mathrm{b}}$ & $1.93 \pm 0.03^{\mathrm{a}}$ & $0.08 \pm 0.04$ & $0.84 \pm 0.02^{\mathrm{a}}$ & $3.41 \pm 0.03^{b}$ \\
\hline & V1 & $5.38 \pm 0.23^{c}$ & $1.65 \pm 0.13^{\mathrm{a}}$ & $0.07 \pm 0.02$ & $0.77 \pm 0.01^{\mathrm{a}}$ & $3.37 \pm 0.03^{a}$ \\
\hline & V2 & $5.15 \pm 0.44^{\mathrm{bc}}$ & $1.73 \pm 0.10^{\mathrm{a}}$ & $0.03 \pm 0.01$ & $0.79 \pm 0.01^{\mathrm{a}}$ & $3.47 \pm 0.03^{b}$ \\
\hline & V3 & $4.87 \pm 0.09^{b}$ & $1.95 \pm 0.05^{b}$ & $0.02 \pm 0.00$ & $0.84 \pm 0.02^{b}$ & $3.61 \pm 0.05^{c}$ \\
\hline & V4 & $4.10 \pm 0.16^{\mathrm{a}}$ & $2.00 \pm 0.07^{b}$ & $0.11 \pm 0.07$ & $0.88 \pm 0.02^{\mathrm{c}}$ & $3.59 \pm 0.15^{c}$ \\
\hline C1 & V1 & $5.75 \pm 0.33^{\mathrm{a}}$ & $1.40 \pm 0.12^{\mathrm{a}}$ & $0.04 \pm 0.00$ & $0.77 \pm 0.01$ & $3.31 \pm 0.04^{\mathrm{a}}$ \\
\hline $\mathrm{C} 1$ & V2 & $6.10 \pm 0.06^{\mathrm{a}}$ & $1.53 \pm 0.03^{\mathrm{a}}$ & $0.04 \pm 0.01$ & $0.78 \pm 0.02$ & $3.50 \pm 0.02^{b}$ \\
\hline C1 & V3 & $4.80 \pm 0.17^{b}$ & $1.93 \pm 0.09^{b}$ & $0.02 \pm 0.00$ & $0.81 \pm 0.02$ & $3.71 \pm 0.03^{\mathrm{c}}$ \\
\hline C1 & V4 & $4.33 \pm 0.22^{c}$ & $2.10 \pm 0.12^{\mathrm{b}}$ & $0.03 \pm 0.01$ & $0.84 \pm 0.02$ & $3.93 \pm 0.04^{c}$ \\
\hline $\mathrm{C} 2$ & V1 & $5.00 \pm 0.06^{b}$ & $1.90 \pm 0.06^{b}$ & $0.10 \pm 0.02$ & $0.76 \pm 0.01$ & $3.43 \pm 0.02^{b}$ \\
\hline $\mathrm{C} 2$ & V2 & $4.20 \pm 0.23^{c}$ & $1.93 \pm 0.09^{b}$ & $0.03 \pm 0.01$ & $0.81 \pm 0.02$ & $3.44 \pm 0.05^{b}$ \\
\hline $\mathrm{C} 2$ & V3 & $4.93 \pm 0.09^{b}$ & $1.97 \pm 0.07^{b}$ & $0.02 \pm 0.01$ & $0.86 \pm 0.02$ & $3.50 \pm 0.05^{c}$ \\
\hline $\mathrm{C} 2$ & V4 & $3.87 \pm 0.18^{\mathrm{c}}$ & $1.90 \pm 0.06^{\mathrm{b}}$ & $0.18 \pm 0.14$ & $0.92 \pm 0.02$ & $3.26 \pm 0.07^{\mathrm{a}}$ \\
\hline
\end{tabular}

C1: chitosan oligosaccharide (COS) supplemented at $100 \mathrm{mg} / \mathrm{kg}, \mathrm{C} 2:$ COS supplemented at $150 \mathrm{mg} / \mathrm{kg}, \mathrm{V} 1: \mathrm{valine}$ supplemented at $0.57 \%$, V2: valine supplemented at $0.72 \%$, V3: valine supplemented at $0.87 \%$, V4: valine supplemented at $1.02 \%$ of basal diet, $\mathrm{T}_{3}$ : triiodothyronine, $\mathrm{T}_{4}$ : thyroxine, $\mathrm{TSH}$ : thyroid stimulating hormone, IGF-1: insulin-like growth factor 1

${ }^{\mathrm{a}-\mathrm{c}}$ Within an effect on each response variable, values with a common superscript were not different at $P=0.05$

Table 6 Dry matter, crude protein content, crude fat, ash content, $\mathrm{pH}$, and moisture content of breast meat of broilers of 42-day old broilers when supplemented with various levels of chitosan oligosaccharide and valine

\begin{tabular}{|c|c|c|c|c|c|c|c|}
\hline \multirow{2}{*}{\multicolumn{2}{|c|}{ Treatments }} & \multicolumn{6}{|c|}{ Response variables } \\
\hline & & \multirow{2}{*}{$\begin{array}{l}\text { Dry matter, \% } \\
28.61 \pm 0.27\end{array}$} & \multirow{2}{*}{$\begin{array}{c}\text { Crude protein, \% } \\
84.21 \pm 0.55\end{array}$} & \multirow{2}{*}{$\begin{array}{l}\text { Crude fat, \% } \\
7.48 \pm 0.09^{a}\end{array}$} & \multirow{2}{*}{$\begin{array}{c}\text { Ash, \% } \\
4.60 \pm 0.16^{b}\end{array}$} & \multirow{2}{*}{$\begin{array}{c}\mathrm{pH} \\
5.84 \pm 0.04\end{array}$} & \multirow{2}{*}{$\begin{array}{l}\text { Moisture, \% } \\
71.39 \pm 0.28\end{array}$} \\
\hline $\mathrm{C} 1$ & & & & & & & \\
\hline \multirow[t]{5}{*}{$\mathrm{C} 2$} & & $28.72 \pm 0.21$ & $84.70 \pm 0.38$ & $7.20 \pm 0.18^{b}$ & $5.11 \pm 0.09^{a}$ & $5.83 \pm 0.04$ & $71.28 \pm 0.34$ \\
\hline & $\mathrm{V} 1$ & $27.97 \pm 0.24^{a}$ & $83.38 \pm 0.86$ & $7.93 \pm 0.10^{c}$ & $4.34 \pm 0.19^{a}$ & $5.93 \pm 0.04$ & $72.04 \pm 0.22^{a}$ \\
\hline & V2 & $28.05 \pm 0.21^{\mathrm{a}}$ & $84.16 \pm 0.51$ & $7.45 \pm 0.15^{b}$ & $4.77 \pm 0.13^{b}$ & $5.87 \pm 0.03$ & $71.96 \pm 0.24^{a}$ \\
\hline & V3 & $29.10 \pm 0.17^{b}$ & $84.48 \pm 0.51$ & $7.00 \pm 0.18^{a}$ & $4.95 \pm 0.16^{b}$ & $5.80 \pm 0.03$ & $70.90 \pm 0.40^{b}$ \\
\hline & V4 & $29.56 \pm 0.13^{b}$ & $85.80 \pm 0.39$ & $6.98 \pm 0.09^{a}$ & $5.37 \pm 0.06^{c}$ & $5.74 \pm 0.07$ & $70.44 \pm 0.36^{\mathrm{b}}$ \\
\hline $\mathrm{C} 1$ & V1 & $27.78 \pm 0.48$ & $82.72 \pm 1.63$ & $7.80 \pm 0.12^{c}$ & $3.95 \pm 0.12^{\mathrm{a}}$ & $5.92 \pm 0.04$ & $72.22 \pm 0.28$ \\
\hline $\mathrm{C} 1$ & V2 & $27.97 \pm 0.44$ & $83.83 \pm 0.59$ & $7.65 \pm 0.12^{b}$ & $4.50 \pm 0.06^{b}$ & $5.89 \pm 0.02$ & $72.03 \pm 0.08$ \\
\hline $\mathrm{C} 1$ & V3 & $29.28 \pm 0.22$ & $84.04 \pm 0.05$ & $7.35 \pm 0.06^{a}$ & $4.60 \pm 0.07^{b}$ & $5.81 \pm 0.03$ & $70.72 \pm 0.10$ \\
\hline $\mathrm{C} 1$ & V4 & $29.42 \pm 0.18$ & $86.24 \pm 0.66$ & $7.10 \pm 0.09^{a}$ & $5.35 \pm 0.12^{c}$ & $5.72 \pm 0.13$ & $70.58 \pm 0.39$ \\
\hline $\mathrm{C} 2$ & $\mathrm{~V}_{1}$ & $28.15 \pm 0.17$ & $84.04 \pm 0.77$ & $8.05 \pm 0.14^{c}$ & $4.72 \pm 0.10^{\mathrm{a}}$ & $5.94 \pm 0.09$ & $71.85 \pm 0.38$ \\
\hline $\mathrm{C} 2$ & V2 & $28.12 \pm 0.13$ & $84.48 \pm 0.91$ & $7.25 \pm 0.25^{b}$ & $5.03 \pm 0.07^{b}$ & $5.85 \pm 0.06$ & $71.88 \pm 0.53$ \\
\hline $\mathrm{C} 2$ & V3 & $28.92 \pm 0.24$ & $84.92 \pm 1.05$ & $6.65 \pm 0.17^{a}$ & $5.30 \pm 0.09^{b}$ & $5.79 \pm 0.06$ & $71.08 \pm 0.88$ \\
\hline $\mathrm{C} 2$ & V4 & $29.70 \pm 0.17$ & $85.36 \pm 0.35$ & $6.85 \pm 0.14^{\mathrm{a}}$ & $5.39 \pm 0.08^{c}$ & $5.75 \pm 0.06$ & $70.30 \pm 0.69$ \\
\hline
\end{tabular}

C1: chitosan oligosaccharide (COS) supplemented at $100 \mathrm{mg} / \mathrm{kg}, \mathrm{C} 2$ : COS supplemented at $150 \mathrm{mg} / \mathrm{kg}$, V1: valine supplemented at $0.57 \%$, V2: valine supplemented at $0.72 \%$, V3: valine supplemented at $0.87 \%$, V4: valine supplemented at $1.02 \%$ of basal diet

${ }_{\mathrm{a}-\mathrm{c}}$ Within an effect on each response variable, values with a common superscript were not different at $P=0.05$ 
The interaction between the supplement levels was significant for percentage ash and $\mathrm{pH}(P<0.05)$. The differences among the treatments for $\mathrm{pH}$ were of little practical consequence. However, percentage ash increased with the level of supplemental Val at $\mathrm{C} 1$ and decreased with augmented levels of supplemental $\mathrm{Val}$ at C2. The contents of dry matter, crude protein, crude fat and moisture were all affected by the level of Val supplementation $(P<0.05)$, but only crude fat was affected $(P<0.01)$ by the level of COS in the diet. Increasing the level of dietary $\mathrm{Val}$ produced drier meat from the leg of the broilers. The leg meat also contained more protein and less fat. The crude fat content of the leg meat was reduced by increasing the level of COS supplementation from C1 to C2. Proximate composition of leg meat as presented in Table 7.

Table 7 Dry matter, crude protein content, crude fat, ash content, $\mathrm{pH}$, and moisture content of leg meat of broilers of broilers at 42 days old when supplemented with various levels of chitosan oligosaccharide and valine

\begin{tabular}{|c|c|c|c|c|c|c|c|}
\hline \multirow{2}{*}{\multicolumn{2}{|c|}{ Treatments }} & \multicolumn{6}{|c|}{ Response variables } \\
\hline & & \multirow{2}{*}{$\begin{array}{c}\text { Dry matter, \% } \\
28.32 \pm 0.52\end{array}$} & Crude protein, \% & \multirow{2}{*}{$\begin{array}{l}\text { Crude fat, \% } \\
15.14 \pm 0.16^{\mathrm{a}}\end{array}$} & \multirow{2}{*}{$\begin{array}{c}\text { Ash, \% } \\
4.15 \pm 0.10^{b}\end{array}$} & \multirow{2}{*}{$\begin{array}{c}\mathrm{pH} \\
5.71 \pm 0.03\end{array}$} & \multirow{2}{*}{$\begin{array}{l}\text { Moisture, \% } \\
71.68 \pm 0.48\end{array}$} \\
\hline $\mathrm{C} 1$ & & & $71.44 \pm 0.57$ & & & & \\
\hline \multirow[t]{5}{*}{$\mathrm{C} 2$} & & $28.77 \pm 0.43$ & $71.94 \pm 0.45$ & $14.66 \pm 0.18^{b}$ & $4.37 \pm 0.12^{\mathrm{a}}$ & $5.73 \pm 0.03$ & $71.23 \pm 0.44$ \\
\hline & V1 & $26.70 \pm 0.40^{\mathrm{a}}$ & $70.62 \pm 0.61^{a}$ & $15.70 \pm 0.15^{b}$ & $4.29 \pm 0.19^{b c}$ & $5.83 \pm 0.02^{c}$ & $73.31 \pm 0.13^{\mathrm{c}}$ \\
\hline & V2 & $27.82 \pm 0.37^{\mathrm{a}}$ & $71.04 \pm 0.41^{\mathrm{ab}}$ & $14.78 \pm 0.20^{\mathrm{a}}$ & $4.36 \pm 0.21^{\mathrm{c}}$ & $5.74 \pm 0.02^{b}$ & $72.18 \pm 0.44^{b}$ \\
\hline & V3 & $29.42 \pm 0.31^{b}$ & $72.38 \pm 0.47^{b c}$ & $14.68 \pm 0.22^{\mathrm{a}}$ & $4.16 \pm 0.03^{\mathrm{a}}$ & $5.70 \pm 0.01^{b}$ & $70.58 \pm 0.34^{a}$ \\
\hline & V4 & $30.24 \pm 0.38^{b}$ & $72.71 \pm 0.41^{\mathrm{C}}$ & $14.45 \pm 0.07^{\mathrm{a}}$ & $4.25 \pm 0.18^{b}$ & $5.60 \pm 0.03^{\mathrm{a}}$ & $69.76 \pm 0.22^{a}$ \\
\hline $\mathrm{C} 1$ & V1 & $26.44 \pm 0.54$ & $70.18 \pm 0.62$ & $15.80 \pm 0.68$ & $3.87 \pm 0.01^{\mathrm{bc}}$ & $5.80 \pm 0.03^{c}$ & $73.56 \pm 0.15$ \\
\hline $\mathrm{C} 1$ & V2 & $27.28 \pm 0.62$ & $70.80 \pm 0.61$ & $15.15 \pm 0.29$ & $3.88 \pm 0.01^{\mathrm{c}}$ & $5.78 \pm 0.01^{b}$ & $72.72 \pm 0.22$ \\
\hline $\mathrm{C} 1$ & V3 & $29.51 \pm 0.64$ & $72.16 \pm 0.38$ & $15.05 \pm 0.17$ & $4.20 \pm 0.06^{a}$ & $5.70 \pm 0.03^{b}$ & $70.49 \pm 0.58$ \\
\hline $\mathrm{C} 1$ & V4 & $30.04 \pm 0.66$ & $72.60 \pm 0.61$ & $14.55 \pm 0.20$ & $4.64 \pm 0.03^{b}$ & $5.55 \pm 0.03^{\mathrm{a}}$ & $69.96 \pm 0.31$ \\
\hline $\mathrm{C} 2$ & $\mathrm{~V}_{1}$ & $26.95 \pm 0.66$ & $71.06 \pm 1.13$ & $15.60 \pm 0.12$ & $4.70 \pm 0.03^{\mathrm{bc}}$ & $5.85 \pm 0.03^{c}$ & $73.05 \pm 0.06$ \\
\hline $\mathrm{C} 2$ & V2 & $28.36 \pm 0.09$ & $71.28 \pm 0.65$ & $14.40 \pm 0.12$ & $4.83 \pm 0.05^{\mathrm{c}}$ & $5.70 \pm 0.03^{b}$ & $71.64 \pm 0.80$ \\
\hline $\mathrm{C} 2$ & V3 & $29.33 \pm 0.25$ & $72.60 \pm 0.95$ & $14.30 \pm 0.17$ & $4.11 \pm 0.02^{\mathrm{a}}$ & $5.70 \pm 0.01^{b}$ & $70.67 \pm 0.48$ \\
\hline $\mathrm{C} 2$ & V4 & $30.44 \pm 0.51$ & $72.82 \pm 0.62$ & $14.35 \pm 0.23$ & $3.85 \pm 0.03^{b}$ & $5.65 \pm 0.03^{\mathrm{a}}$ & $69.56 \pm 0.31$ \\
\hline \multicolumn{4}{|c|}{ Source of variation } & $P$-value & & & \\
\hline \multicolumn{2}{|c|}{$\cos$} & \multicolumn{2}{|c|}{0.250} & 0.002 & $<0.0001$ & 0.365 & 0.157 \\
\hline \multicolumn{2}{|l|}{ Val } & \multicolumn{2}{|c|}{$<0.0001$} & $<0.0001$ & $<0.0001$ & $<0.0001$ & $<0.0001$ \\
\hline \multicolumn{2}{|c|}{$\mathrm{COS}^{*} \mathrm{Val}$} & \multicolumn{2}{|c|}{0.712} & 0.251 & $<0.0001$ & 0.023 & 0.555 \\
\hline
\end{tabular}

C1: chitosan oligosaccharide (COS) supplemented at $100 \mathrm{mg} / \mathrm{kg}, \mathrm{C} 2$ : COS supplemented at $150 \mathrm{mg} / \mathrm{kg}$, V1: valine supplemented at $0.57 \%$, V2: valine supplemented at $0.72 \%$, V3: valine supplemented at $0.87 \%$, V4: valine supplemented at $1.02 \%$ of basal diet

The increase in live BW and WG in the groups confirmed COS as a prebiotic and that Val had a growth-promoting effect on broilers by improving muscle protein synthesis and nutrient digestibility and by influencing the activity and composition of gut microbiota. An increase in FI showed that supplementation of $\mathrm{COS}$ and Val could improve nutrient digestibility and consequently feed efficiency and growth performance. $A$ reduction in FCR made it evident that $\mathrm{C} 1$ and $\mathrm{C} 2$ could lower FCR and improve growth performance in broilers and in other poultry. Corzo et al. (2010); Lee et al. (2014), and Bednarczyk et al. (2016) also found growth-promoting influence with Bacillus subtilis, prebiotic, isoleucine, and Val supplementation in broilers.

Dietary supplementations of COS and Val resulted in a linear increase in carcass weight and carcass percentage, which was expected from the rise in live BW and WG. A linear reduction in abdominal fat with increasing levels of COS and Val revealed that supplementation is hypolipidemic and suppresses the fat deposition in adipocytes. It was suggested that the decrease in abdominal fat was because of lowered synthesis of mRNA (Foretz et al., 1999; Amirdahri et al., 2020) for rate-limiting enzymes in hepatic tissues, decreasing the fatty acid synthesis. Alternatively, supplementation possibly increased the level of mRNA for rate-limiting enzymes in hepatic tissues, increasing fatty acid oxidation, which is mediated through the 
regulation of the transcription of the sterol regulatory element binding protein-1c (SREBP-1C). However, no effect was observed on relative weights of liver, heart, spleen, gizzard and gall bladder (Abeer \& Mosaad, 2015; Abdel-Hafeez et al., 2017).

The increase in thyroid hormones $\left(T_{3}\right.$ and $\left.T_{4}\right)$ may have been due to greater release of hypothalamic $\mathrm{TRH}$, which stimulated the secretion of TSH from adenohypophysis, eventually promoting $\mathrm{T}_{3}$ and $\mathrm{T}_{4}$ synthesis and release. The linear and interactive effects in the levels of testosterone and IGF-1 indicated that the supplementation of amino acids had a growth-promoting effect on broilers, which may have implications for other poultry. Increased levels of $T_{3}, T_{4}$, testosterone, and IGF-1 influence the metabolism and may ultimately affect the performance, carcass and abdominal fat percentages. Buyse et al. (1992), Zhai et al. (2016) and lbitoye et al. (2019) described similar findings resulting from supplementation of lysine at $100 \%$ to $120 \%$ of recommended levels and chitosan at $0.5 \mathrm{~g} / \mathrm{kg}$ in broiler diets.

The proximate composition of breast and leg meat samples exhibited a non-significant increase in dry matter and crude protein because both amino acid and prebiotic supplementation could increase muscle weight and consequently the dry matter in the of broilers. It is generally recommended that healthy broiler meat should contain a higher proportion of protein, a lower content of fat, and adequate minerals. However, the decrease in crude fat content revealed that supplements can lessen the crude fat content in meat of broilers by inhibiting the deposition of fats (Valsta et al., 2005; Abdurrahman et al., 2016). Murakami et al. (2010) and Ferreira et al. (2015) also conducted studies and reported decreased crude fat content in meat composition of broilers supplemented with linseed oil and Val.

Moreover, the increment in ash content, a parameter to estimate the mineral amount of a foodstuff, was evident from greater crude protein content in the samples. However, the decreased $\mathrm{pH}$ and moisture content in breast and leg meat samples with the enhanced concentration of supplements was expected as a result of greater content of dry matter. Erwan et al. (2009) studied the composition of broiler meat and its sensory characteristics supplemented with varying levels of leucine and reported similar changes in $\mathrm{pH}$, which affected its moisture content and water-holding capacity of meat and physical qualitative traits, including tenderness, colour and moisture absorption (Husak et al., 2008).

\section{Conclusions}

Dietary inclusion of C2 and V3 to V4 improved growth performance, carcass characteristics, and hormonal profiles. These findings suggest that a dietary formulation for farmers that contained these supplements would improve the meat quantitatively and qualitatively.

\section{Acknowledgements}

The contribution of Animal Nutrition Research Laboratory, UVAS, Pattoki Campus, Pakistan is greatly acknowledged for providing facility and guidance to analyse the meat quality attributes.

\section{Authors' Contributions}

IA (ORCID: 0000-0002-9858-5064) executed the experiment, statistically analysed the collected data and completed the manuscript; NR (OCID: 0000-0002-2396-5433) critically analysed, reviewed and supported in final compilation of manuscript; and AR (ORCID: 0000-0003-1737-7843) helped in preparing and drafting the manuscript.

\section{Conflict of Interest Declaration}

The authors have no financial or other association with persons or organizations that could have inappropriate influence on this paper or bias the contents of this research article.

\section{References}

Abdel-Hafeez, H.M., Saleh, E.S.E. \& Tawfeek, S.S., 2017. Effects of probiotic, prebiotic, and symbiotic with and without feed restriction on performance, hematological indices and carcass \& characteristics of broiler chickens. Asian. Aust. J. Anim. Sci. 30(5), 672-682. DOI 10.5713/ajas.16.0535

Abdurrahman, Z.H., Pramono, Y.B. \& Suthama, N., 2016. Meat characteristic of crossbred local chicken fed inulin of dahlia tuber and lactobacillus sp. Media. Peternakan. 39(2), 112-118. DOI: 10.5398/medpet.2016.39.2.112

Abeer, E.S.M. \& Mosaad, S.A., 2015. Effect of dietary probiotic and/or prebiotic supplementation on growth performance, carcass traits and some serum biochemical alterations in broiler chicken. J. Anim. Sci. Adv. 5(11), 1480-1492.

Aftab, U., 2009. Response of broilers to practical diets with different metabolizable energy and balanced protein concentrations. Braz. J. Poult. Sci.11(3), 169-173. DOI: 10.1590/S1516-635X2009000300005

Amirdahri, S., Janmohammadi, H., Lambert, W., Soumeh, E. A. \& Oliayi, M., 2020. Valine requirement of female Cobb broilers from 8 to 21 days of age. 2020 J. Appl. Poult. Res. DOI 10.1016/j.japr.2020.04.005

AOAC, 2006. Official methods of analysis. 18th ed. Association of Official Analytical Chemists, Arlington, VA.

Ayasan, T. \& Okan, F., 2014. The effect of choice feeding based on threonine on performance and carcass parameters of male broiler chicks. Turkish Journal of Agriculture, Food Science and Technology. 2(4),190-196. DOI: 10.24925/turjaf.v2i4.190-196.117 
Ayasan, T., Okan, F. \& Hizli, H., 2009. Threonine requirement of broiler from 22 to 42 days. Int. J. Poult. Sci. 8 (9), $862-$ 865. DOI: 10.3923/ijps.2009.862.865

Bednarczyk, M., Stadnicka, K., Kozłowska, I., Abiuso, C., Tavaniello, S., Dankowiakowska, A. \& Maiorano, G., 2016. Influence of different prebiotics and mode of their administration on broiler chicken performance. Animal 10(8), 1271-1279. DOI: $10.1017 / \mathrm{S} 1751731116000173$

Buyse, J., Decuypere, E., Berghman, L., Kuhn, E.R. \& Vandesande, F., 1992. Effect of dietary protein content on episodic growth hormone secretion and on heat production of male broiler chickens. Brit. Poult. Sci. 33(5), 1101 1109. DOI: $10.1080 / 00071669208417552$

Corzo, A., Dozier III, W.A., Loar, R.E., Kidd, M.T. \& Tillman, P.B., 2010. Dietary limitation of isoleucine and Valine in diets based on maize, soybean meal, and meat and bone meal for broiler chickens. Br. Poult. Sci. 51(4), 558563.DOI:10.1080/00071668.2010.507242

Duncan, D.B., 1955. Multiple range and multiple F tests. Biometrics 11, 1-42.

Duarte, K.F., Junqueira, O.M., Domingues, C.H.D.F., Filardi, R.D.S., Borges, L.L. \& Praes, M.F.F.M., 2014. Digestible valine requirements for broilers from 22 and 42 days old. Acta Sci. Anim. Sci. 36(2), 151-156. DOI: 10.4025/actascianimsci.v36i2.22761

Erwan, E., Alimon, A.R., Sazili, A.Q., Yaakub, H. \& Karim, R., 2009. Effects of varying levels of L-leucine and metabolizable energy in finisher diet on carcass composition and meat sensory characteristics of broiler chickens. Pak. J. Nutr. 8(6), 792-796.

Ferreira, D.G., Pinto, M.F., Neto, M.G., Ponsano, E.H.G., Goncalves, C.A., Bossolani, I.L.C. \& Pereira, A.G., 2015. Accurate adjustment of energy level in broiler chicken diet for controlling the performance and the lipid composition of meat. Cienc. Rural 45(1),104-110. DOI: 10.1590/0103-8478cr20130206

Ferreira, N.T., de Albuquerque, R., Sakomura, N.K., de Paula Dorigam, J.C., da Silva, E.P., Burbarelli, M.F.C. \& Gous, R. M., 2016. The response of broilers during three periods of growth to dietary Valine. Anim. Feed. Sci. Technol. 214(2016),110-120. DOI: 10.1016/j.anifeedsci.2016.02.017

Foretz, M., Guichard, C., Ferré, P. \& Foufelle, F., 1999. Sterol regulatory element binding protein-1c is a major mediator of insulin action on the hepatic expression of glucokinase and lipogenesis-related genes. Proceedings of the National Academy of Sciences, 96(22), 12737-12742. DOI: 10.1073/pnas.96.22.12737

Haque, M.I., Ahmad, N. \& Miah, M.A., 2017. Comparative analysis of body weight and serum biochemistry in broilers supplemented with some selected probiotics and antibiotic growth promoters. J. Adv. Vet. Anim. Res. 4(3), 288294. DOI: 10.5455/javar.2017.d226

Husak, R.L., Sebranek, J.G. \& Bregendahl, K., 2008. A survey of commercially available broilers marketed as organic, free-range, and conventional broilers for cooked meat yields, meat composition, and relative value. Poult. Sci. 87(11), 2367-2376. DOI: 10.3382/ps.2007-00294

Ibitoye, E.B., Lokman, I.H., Hezmee, M.N.M., Goh, Y.M., Zuki, A.B.Z., Jimoh, A.A. \& Nicholas, N.P., 2019. Gut health and serum growth hormone levels of broiler chickens fed dietary chitin and chitosan from cricket and shrimp. Poultry Science, 98(2), 745-752. DOI: 10.3382/ps/pey419

Janardhana, V., Broadway, M.M., Bruce, M.P., Lowenthal, J.W., Geier, M.S., Hughes, R.H. \& Bean, A.G., 2009. Prebiotics modulate immune responses in the gut-associated lymphoid tissue of chickens. J. Nutr. 139(7), 14041409. DOI: $10.3945 /$ jn.109.105007

Lee, S.H., Ingale, S.L., Kim, J.S., Kim, K.H., Lokhande, A., Kim, E.K. \& Chae, B.J., 2014. Effects of dietary supplementation with Bacillus subtilis LS 1-2 fermentation biomass on growth performance, nutrient digestibility, cecal microbiota and intestinal morphology of weanling pig. Anim. Feed. Sci. Tech. 188(2014), 102-110. DOI: 10.1016/j.anifeedsci.2013.12.001

Li, X.J., Piao, X.S., Kim, S.W., Liu, P., Wang, L., Shen, Y.B. \& Lee, H.S., 2007. Effects of chito-oligosaccharide supplementation on performance, nutrient digestibility, and serum composition in broiler chickens. Poult. Sci. 86(6), 1107-1114. DOI: 10.1093/ps/86.6.1107

Liu, P., Piao, X.S., Kim, S.W., Wang, L., Shen, Y.B., Lee, H.S. \& Li, S.Y., 2008. Effects of chito-oligosaccharide supplementation on the growth performance, nutrient digestibility, intestinal morphology, and fecal shedding of Escherichia coli and Lactobacillus in weaning pigs. J. Anim. Sci. 86(10), 2609-2618. DOI: 10.2527/jas.2007-0668

Liu, W., Yuan, Y., Sun, C., Balasubramanian, B., Zhao, Z. \& An, L., 2019. Effects of dietary betaine on growth performance, digestive function, carcass traits, and meat quality in indigenous yellow-feathered broilers under long-term heat stress. Animals 9(8), 506. DOI: 10.3390/ani9080506

Miranda, D.J.A., Vieira, S.L., Favero, A., Angel, C.R., Stefanello, C. \& Nogueira, E.T., 2015. Performance and meat production of broiler chickens fed diets formulated at different crude protein levels supplemented or not with 1 Valine and I-isoleucine. Anim. Feed. Sci. Technol. 206(2015), 39-47. DOI: 10.1016/j.anifeedsci.2015.04.018

Murakami, K.T.T., Pinto, M.F., Ponsano, E.H.G. \& Garcia, N., 2010. Yield performance and meat quality of broilers fed diets with linseed oil. Pesq. Agropec. Bras. 45, 401-407. DOI: 10.1590/S0100-204X2010000400008

Nazir, A., Raza, A., Akbar, A., Ahmad, A., Aziz, A., Iqbal, M., Arooj, T. \& Ehtisham-ul-Haque, S., 2020. Novel approach for investigation of antibiotic residue in broilers grown under different agro-ecological conditions. Polish Journal of Environmental Studies 29(6). DOI 10.15244/pjoes/115174

Ospina-Rojas, I.C., Pozza, P.C., Rodrigueiro, R.J.B., Gasparino, E., Khatlab, A.S. \& Murakami, A.E., 2020. High leucine levels affecting valine and isoleucine recommendations in low-protein diets for broiler chickens. Poult. Sci. \&99(11), 5946-5959. DOI: 10.1016/j.psj.2020.08.053

Ospina-Rojas, I.C., Murakami, A.E., Duarte, C.R.A., Eyng, C., Oliveira, C.A.L. \& Janeiro, V., 2014. Valine, isoleucine, arginine and glycine supplementation of low-protein diets for broiler chickens during the starter and grower phases. Br. Poult. Sci. 55(6), 766-773. DOI: 10.1080/00071668.2014.970125 
Qu, H., Cheng, Y., Chen, Y., Li, J., Zhao, Y. \& Zhou, Y., 2019. Effects of dietary zeolite supplementation as an antibiotic alternative on growth performance, intestinal integrity, and cecal antibiotic resistance genes abundances of broilers. Animals 9(11), 909. DOI: 10.3390/ani9110909

Qureshi, M.A., Heggen, C.L. \& Hussain, I., 2000. Avian macrophage: Effector functions in health and disease. Dev. Comp. Immunol. 24, 103-119. DOI: 10.1016/s0145-305x(99)00067-1

Rostagno, H.S., Albino, L.F.T., Donzele, J.L., Gomes, P.C., Oliveira, R.F.M., Lopes, D.C., Ferreira, A.S. \& Barreto, S.L.T., 2011. Brazilian tables for poultry and swine: Composition of feedstuffs and nutritional requirements. 3rd ed. Vicosa MG, Brasil.

Sedghi, M., Golian, A., Kolahan, F. \& Afsar, A., 2015. Optimization of broiler chicken responses from 0 to 7 days of age to dietary leucine, iso-leucine and valine using Taguchi and mathematical methods. Br. Poult. Sci. 56(6), 696-707. DOI: 10.1080/00071668.2015.1096323

Shi, B.L., Li, D.F., Piao, X.S. \& Yan, S.M. 2005. Effects of chitosan on growth performance and energy and protein utilization in broiler chickens. Brit. Poultry Sci. 46(2005): 516-519. DOI: 10.1080/00071660500190785

Sterling, K.G., Pesti, G.M. \& Bakalli, R.I., 2003. Performance of broiler chicks fed various levels of dietary lysine and crude protein. Poultry Science 82(12), 1939-1947. DOI: 10.1093/ps/82.12.1939

Swiatkiewicz, S., Swiatkiewicz, M., Arczewska-Wlosek, A. \& Jozefiak, D., 2015. Chitosan and its oligosaccharide derivatives (chito-oligosaccharides) as feed supplements in poultry and swine nutrition. J. Anim. Physiol. Anim. Nutr. 99(1), 1-12. DOI 10.1111/jpn.12222

Valsta. L.M., Tapanainen, H. \& Mannisto, S., 2005. Meat fats in nutrition. Meat Sci 70:525-530.

Xiong, X., Yang, H.S., Wang, X.C., Hu, Q., Liu, C.X., Wu, X., Deng, D., Hou, Y.Q., Nyachoti, C.M., Xiao D.F. \& Yin, Y.L., 2015. Effect of low dosage of chito-oligosaccharide supplementation on intestinal morphology, immune response, antioxidant capacity, and barrier function in weaned piglets. J. Anim. Sci. 93(3), 1089-97. DOI 10.2527/jas.20147851

Zhai, W., Peebles, E.D., Wang, X., Gerard, P.D., Olanrewaju, H.A. \& Mercier, Y., 2016. Effects of dietary lysine and methionine supplementation on Ross 708 male broilers from 21 to $42 \mathrm{~d}$ of age (III): serum metabolites, hormones, and their relationship with growth performance. J. App. Poult.Res.; 25(2), 223-231. DOI 10.3382/japr/pfw004

Zhao, Z., Li, S., Huang, H., Li, C., Wang, Q. \& Xue, L., 2015. Comparative study on growth and developmental model of indigenous chicken breeds in China. Open. J. Anim. Sci. 5(02), 219-223. DOI: 10.4236/ojas.2015.52024 